\title{
The Completeness of Carnap's Predicate Logic
}

\author{
M.J. Cresswell \\ Victoria University of Wellington \\ max.cresswell@msor.vuw.ac.nz
}

\begin{abstract}
The paper first proves the completeness of the (non-modal) first-order predicate logic presented in Carnap's 1946 article 'Modalities and quantification'. By contrast the modal logic defined by the semantics Carnap produces is unaxiomatisable. One can though adapt Carnap's semantics so that a standard completeness proof for a Carnapian version of predicate S5 turns out to be available.
\end{abstract}

The purpose of this note is to prove the completeness of the non-modal predicate logic found in Carnap $1946^{1}$, and of a Carnapian version of predicate S5, with respect to a semantics a little different from Carnap's. I will say at the outset that the results and proof methods used are not surprising or significantly innovative; and the reason for the note is simply that, as far as I am aware, no one has proved specifically the completeness of Carnap's 1946 predicate logic. In setting this out I shall avoid Carnap's use of German letters, which make his work difficult for current readers. Since Carnap's labels for axioms and so on are specific to his paper I have used names which are more indicative of their content. Further, I shall base my presentation of Carnap's logic on $\perp$ and $\supset$ rather than on Carnap's $\sim$, $\checkmark$ and ' $\therefore$. For quantifiers I use $\forall x$ in place of $(x)$, and $\exists x$ in place of $(\exists x) .^{2}$ Carnap's language of FL (first order logic) contains a denumerable infinity of individual variables and constants, which can be referred to simply as 'variables' or 'constants'. Carnap uses the term 'matrix' for a (well-formed) formula (wff), and the term 'sentence' for a closed wff, i.e., a wff with no free variables. When it comes to interpreting FL Carnap (p. 49) assumes denumerable domains. For this reason he is able to represent a domain by a denumerable set of individual constants - a distinct constant for each individual. The

\footnotetext{
${ }^{1}$ Carnap does not claim to prove completeness, and on p. 53 he says that it is not clear whether his axiom system is complete, since Gödel's completeness theorem (Gödel, 1930) "cannot be directly applied" to it. Carnap's article predates the completeness proof for first-order predicate logic found in Henkin 1949. The present note provides a Henkin proof for an axiomatic system equivalent to Carnap's FC.

${ }^{2}$ The other truth functions are all definable in terms of $\supset$ and $\perp$; in particular $\alpha \vee \beta$ may be defined as $(\alpha \supset \beta) \supset \beta, \sim \alpha$ as $\alpha \supset \perp$ and $\alpha \wedge \beta$ as $\sim(\alpha \supset \sim \beta)$. Carnap takes $\sim, \vee$ and '.' $(\wedge)$ as primitive, but for the points made here these differences do not matter. $\exists x \alpha$ may be defined as $\sim \forall x \sim \alpha$. Carnap does not introduce an explicit definition, but does use $\exists$ on p. 53 .
} 
presence of constants enables there to be atomic sentences like $\varphi a b$. Truth and falsity in FL are relative to a state-description (Carnap 1946, p. 50, and 1947, p. 9). Formally, a state-description $s$ for a language $\mathcal{L}$ of first-order predicate logic is a set of atomic sentences or their negations, such that for every n-place predicate $\varphi$ of $\mathcal{L}$, and every ntuple of constants $a_{1}, \ldots, a_{\mathrm{n}}$, either $\varphi a_{1} \ldots a_{\mathrm{n}} \in s$ or $\sim \varphi a_{1} \ldots a_{\mathrm{n}} \in s$, but not both. In place of relativising the truth of an open formula to an assignment to the variables Carnap defines truth only for sentences. If $s$ is any state-description, I write $s=\alpha$ to mean that $\alpha$ is true in $s$ and $s=\alpha$ to mean that $\alpha$ is false in $s$. The truth of a sentence $\alpha$ in a state-description $s$ may be defined formally by the following valuation rules:

$$
\begin{aligned}
& {[\mathrm{V} \varphi] s \models \varphi a_{1} \ldots a_{\mathrm{n}} \text { if } \varphi a_{1} \ldots a_{\mathrm{n}} \in s, \text { and } s=\varphi \mathrm{a}_{1} \ldots a_{\mathrm{n}} \text { if } \sim \varphi a_{1} \ldots a_{\mathrm{n}} \in s} \\
& {[\mathrm{~V}=] s \models a=b \text { iff } a \text { and } b \text { are the same constant }} \\
& {[\mathrm{V} \perp] s=\perp} \\
& {[\mathrm{V} \supset] s \models \alpha \supset \beta \text { iff } s=\alpha \text { or } s \models \beta} \\
& {[\mathrm{V} \forall] s \models \forall x \alpha \text { iff } s \models \alpha[a / x] \text { for every constant } a, \text { where }[\alpha / x] \text { is } \alpha \text { with } a \text { replacing }} \\
& \quad \text { every free } x .^{3}
\end{aligned}
$$

$s \models \alpha$ means that $\alpha$ is true in $s$. Carnap does not speak of truth in a state-description, but speaks of the range of a sentence - which is a set of state-descriptions. Where I have said $s \models \alpha$, Carnap will say that $s$ is in the range of $\alpha$. Carnap's 'rules of ranges' (p. 50) then become $[\mathrm{V} \varphi]-[\mathrm{V} \forall]$, allowing for the adoption of $\perp$ and $\supset$ as the primitive truth-functional operators. Say that a sentence $\alpha$ is valid, or in Carnap's words $L$-true, iff $s \mid=\alpha$ for every state-description $s$.

Carnap produces an axiomatic basis for what he calls 'FC'. In Carnap's terminology a 'logic' is a semantic specification of a set of formulae, while a 'calculus' is an axiomatic specification. 'FC' stands for 'functional calculus', and I shall use it to refer to the version presented here of Carnap's axiomatic system. Carnap refers to axioms as 'primitive sentences'. In place of Carnap's ( ) to indicate a universal closure of a wff, I shall write $\forall \alpha$. More specifically, where $\alpha$ is any wff, $\forall \alpha$ denotes $\alpha$ preceded by a (finite) sequence of universal quantifiers, in any order, which includes one for every variable free in $\alpha$. (Where $\alpha$ is a sentence then $\alpha$ itself is an instance of $\forall \alpha$.) With that notation Carnap's first axiom schema, D8-1a (p. 52), can be written as:

\footnotetext{
${ }^{3}$ This feature of Carnap's semantics can be thought of as a version of the 'substitutional' interpretation of the quantifiers - to the extent that Leblanc 1973, p. 2, refers to the substitutional account of quantification as 'Carnap's account'. Leblanc 1969 (following Beth 1959, pp. 263-267) provides a completeness proof for a Carnap-like system. What makes the present completeness proof different is the feature of identity reflected in $[\mathrm{V}=]$, that $a=b$ is always false when $a$ and $b$ are distinct constants. This latter feature has some similarities with the interpretation of Wittgenstein's Tractatus-Logico-Philosophicus (Wittgenstein 1922, for instance at 5.53) studied in Rogers and Wehmeier 2012, pp. 540-555, though Wittgenstein appears to suggest that identity statements, like say $a=b$, and even presumably $x=y$, cannot even be stated.
}

Australasian Journal of Logic (11) 2014, Article no. 3 
PC Any wff of the form $\forall \alpha$ where $\alpha$ is a PC-tautology, i.e., where $\alpha$ can be obtained from a valid formula of the classical propositional calculus by uniform substitution of wff of $\mathcal{L}$ for its propositional variables. ${ }^{4}$

and the remaining schemata can be represented as:

$\forall \supset \forall(\forall x(\alpha \supset \beta) \supset(\forall x \alpha \supset \forall x \beta))$

VQ $\forall(\alpha \supset \forall x \alpha)$, where $x$ is not free in $\alpha$.

$\forall \mathbf{1 a} \forall(\forall x \alpha \supset \alpha[y / x])$, where $\alpha[y / x]$ is just like $\alpha$ except in having free $y$ in place of free $x$, where $y$ is any variable for which $x$ is free

$\forall \mathbf{1 b} \forall(\forall x \alpha \supset \alpha[a / x])$, where $\alpha[a / x]$ is just like $\alpha$ except in having $a$ in place of free $x$, where $a$ is any constant

I1 $\forall x x=x$

I2 $\forall(x=y \supset(\alpha \supset \beta))$, where $\alpha$ and $\beta$ are alike except that $\alpha$ has free $x$ in 0 or more places where $\beta$ has free $y .^{5}$

I3 $a \neq b$ where $a$ and $b$ are different constants.

The only transformation rule is modus ponens:

$\mathbf{M P} \vdash \alpha, \vdash \alpha \supset \beta \rightarrow \vdash \beta$

In MP of course, given that $\vdash \alpha$ and $\vdash \alpha \supset \beta$, then $\alpha$ and $\beta$ and $\alpha \supset \beta$ must all be sentences. Carnap's axiomatisation follows that of Quine 1940 in restricting theorems to closed wff. In what follows proofs will be given in outline only, especially where full proofs may be found of these or similar theorems on pp. 80-115 of Quine's book. The principal non-standard feature is I3, which entails that all state descriptions are infinite, and, as Carnap points out on p. 53, sentences like $\exists x \exists y \quad x \neq y$ are true in universes containing more than one individual.

\footnotetext{
${ }^{4}$ This is slightly more general than the definition Carnap uses, since it requires that the $\forall$ s in instances of $\forall \alpha$, where $\alpha$ is an instance of a PC tautology, include all prefixes consisting of universal quantifiers, in any order, whose variables are those free in $\alpha$. In this respect I am following Fitch 1941, commenting on Quine 1940, whose first edition contained an axiom schema stating that the order of two adjacent universal quantifiers makes no logical difference. This issue is a complex one discussed in some detail in Fine 1983. What Carnap says on p. 51 is that " $\left(\right.$ ( $\left(\left[\mathcal{M}_{k}\right]\right)$ ' denotes the closure of $\mathcal{M}_{k}$, i.e. the sentence formed out of $\mathcal{M}_{k}$ by prefixing universal quantifiers for all variables occurring freely in $\mathcal{M}_{k}$, in their inverse alphabetical order." He then (footnote 14, p. 52) cites Berry 1941 as establishing that this is sufficient to enable the proof that the order of a sequence of universal quantifiers is immaterial.

${ }^{5}$ Carnap, D8-1g, (1946, p. 52), appears to require that $\beta$ must have free $y$ wherever $\alpha$ has free $x$, but that seems too stringent, since $\forall x \forall y(x=y \supset(\varphi x x \supset \varphi x y))$ is valid.
}

Australasian Journal of Logic (11) 2014, Article no. 3 
We cannot, in Carnap's basis, appeal to the rule of universal generalisation in the form, $\vdash \alpha \rightarrow \vdash \forall x \alpha$, because Carnap's theorems are all sentences. Nor can we appeal to the rule $\vdash \alpha \rightarrow \vdash \forall x(\alpha[x / a]$ ), (where $\alpha[x / a]$ is $\alpha$ with free $x$ replacing every occurrence of $a$ in $\alpha$ ), since, when $a$ and $b$ are distinct constants we have $\vdash a \neq b$, but we do not have $\vdash$ $\forall x x \neq b .^{6}$ However we can prove the following:

Theorem 1. If $\vdash \alpha$, and $a$ is any constant, then, for some $n \geq 0$, there are constants $b_{1}, \ldots, b_{\mathrm{n}}$ such that $\vdash \forall x\left(\left(x \neq b_{1} \wedge \ldots \wedge x \neq b_{\mathrm{n}}\right) \supset \alpha[x / a]\right)$, provided $x$ does not become bound in $\alpha[x / a]$ as a result of the substitution. (We allow the degenerate case in which there are no bs, so that here $n=0$, and we have simply $\vdash \forall x \alpha[x / a]$.)

Proof. The proof is by induction on the proof of $\alpha$, and depends on the fact that if $\vdash \alpha$ then $\alpha$ is closed. First suppose that $\alpha$ is an instance of one of the axiom schemata PC-I2. Then $\forall x(\alpha[x / a])$ remains an instance of that very same schema. This may be seen by inspection. So for these cases the theorem holds where $n=0$. The remaining case is I3:

$$
\begin{gathered}
\text { I3' } \vdash x(x \neq b \supset x \neq b) \\
\mathbf{I 3}^{\prime \prime} \vdash \forall x(x \neq a \supset a \neq x)
\end{gathered}
$$

$\mathrm{I}^{\prime}$ is an instance of PC, and I3 ${ }^{\prime \prime}$ follows from I1 and I2 using $\forall 1 \mathrm{~b} .^{7}$

Further, MP preserves the rule, since suppose we are given $\vdash \alpha$ and $\vdash \alpha \supset \beta$. Then, by the induction hypothesis, we may assume that there are $b_{1}, \ldots, b_{n}$, and $c_{1}, \ldots, c_{k}$ such that

$$
\text { (i) } \vdash \forall x\left(\left(x \neq b_{1} \wedge \ldots \wedge x \neq b_{n}\right) \supset \alpha[x / a]\right)
$$

and

$$
\text { (ii) } \vdash \forall x\left(\left(x \neq c_{1} \wedge \ldots \wedge x \neq c_{k}\right) \supset(\alpha[x / a] \supset \beta[x / a])\right)
$$

From $(i)$ and $(i i)$, by PC and $\forall^{\supset}$ we have

$$
\text { (iii) } \vdash \forall x\left(\left(x \neq b_{1} \wedge \ldots \wedge x \neq b_{n} \wedge x \neq c_{1} \wedge \ldots \wedge x \neq c_{k}\right) \supset \beta[x / a]\right)
$$

and so the theorem holds for $\beta$.

The next three results are proved in an appendix:

\footnotetext{
${ }^{6}$ Compare Carnap's remarks in T7.2 and T7.3 in Carnap 1946, p. 51f.

${ }^{7}$ This may be proved as follows:

$\mathrm{I} 2 \forall 1 b \quad(1) \quad \forall x(a=x \supset(a=a \supset x=a))$

(1)PC (2) $\forall x(a=a \supset(x \neq a \supset a \neq x))$

(2)I1 (3) $\forall x(x \neq a \supset a \neq x)$
} 
Theorem 2. If $\alpha \supset \beta$ then, for some $b_{1}, \ldots, b_{n}, \vdash \alpha \supset \forall x\left(\left(x \neq b_{1} \wedge \ldots \wedge x \neq b_{n}\right) \supset \beta[x / a]\right)$, provided a does not occur in $\alpha$

Theorem 3. Where $\forall x \alpha$ is a sentence and $b_{1}, \ldots, b_{n}$ are any constants then, $\vdash\left(\alpha\left[b_{1} / x\right] \wedge\right.$ $\left.\ldots \wedge \alpha\left[b_{n} / x\right] \wedge \forall x\left(\left(x \neq b_{1} \wedge \ldots \wedge x \neq b_{n}\right) \supset \alpha\right)\right) \supset \forall x \alpha$

Lemma 4. $\vdash \exists y(\alpha[y / x] \supset \forall x \alpha)$ provided $y$ is not free in $\forall x \alpha$.

In proving soundness we make use of the following lemma:

Lemma 5. Where $s$ is any state-description and $\alpha$ is any wff, where the variables free in $\alpha$ are $x_{1}, \ldots, x_{n}$, and $s \models \alpha\left[a_{1} / x_{1}, \ldots, a_{n} / x_{n}\right]$ for all constants $a_{1}, \ldots, a_{n}$; then $s \models \forall \alpha$.

Theorem 6. If $\vdash \alpha$ then $\alpha$ is L-true

Proof. Soundness may be proved in the usual way, by proving that all axioms are valid and that MP preserves validity. Lemma 5 guarantees that the schematic axioms may be stated, without affecting validity, by leaving off the initial $\forall$, and replacing each distinct free variable by a distinct constant not otherwise occurring, and we shall consider them in this form. Obviously, if $\alpha$ is an instance of a tautology then, for every state-description $s$, $s \models \alpha$. For $\forall^{\supset}$, suppose $s=\forall x(\alpha \supset \beta) \supset(\forall x \alpha \supset \forall x \beta)$. So $s \models \forall x(\alpha \supset \beta), s \models \forall x \alpha$, but $s=\forall x \beta$; and so for some $a, s=\beta[a / x]$. But $s \models \alpha[a / x] \supset \beta[a / x]$, and $s \models \alpha[a / x]$, and so $s \models \beta[a / x]$. For VQ, suppose that $s=\alpha \supset \forall x \alpha$. So $s \models \alpha$ and $s=\forall x \alpha$. But $x$ is not free in $\alpha$, and so $s=\alpha$. For $\forall 1$ a suppose that $s=\forall y(\forall x \alpha \supset \alpha[y / x])$. Then for some $a, s=\forall x \alpha \supset \alpha[a / x]$ and so $s \models \forall x \alpha$ and $s=\alpha[a / x]$ which is a contradiction. This also covers $\forall 1$ b. For I1 $a$ and $a$ are the same constant, and $s=a=a$. For I2, if $s=a=b$ then these are the same constant, and $\alpha$ and $\beta$ are the same sentence, and so $s \mid=\alpha \supset \beta$. Finally if $a$ and $b$ are different constants then $s=a \neq b$. MP preserves validity since if $s \models \alpha$ and $s=\beta$ then $s=\alpha \supset \beta$.

In proving completeness we have to shew that if $s \models \alpha$ for all state-descriptions then $\vdash_{\mathrm{FC}} \alpha$. In fact we would like to get the stronger form of completeness by shewing that if a set $\Lambda$ of sentences is FC-consistent then there is a state-description $s$ such that $s=\alpha$ for every $\alpha \in \Lambda$. At this point we have to bear in mind that the set of state-descriptions is relative to a language $\mathcal{L}$. For take the set $\Omega=\left\{\sim \forall x \varphi x, \varphi a_{1}, \ldots, \varphi a_{n}, \ldots\right\}$ where $\varphi a_{\mathrm{i}} \in$ $\Omega$ for every constant $a_{\mathrm{i}}$ in $\mathcal{L}$. This set is not true in any state-description for $\mathcal{L}$. We can, however, extend $\mathcal{L}$ to a language $\mathcal{L}{ }^{+}$by adding a denumerable infinity of new constants not in $\mathcal{L}$, so that $\Omega$ is satisfiable in $\mathcal{L}{ }^{+}$, though there will be a new $\Omega^{+}$unsatisfiable in $\mathcal{L}^{+}$. This means that where $\Lambda$ is a consistent set of sentences of a language $\mathcal{L}$ then $\Lambda$ may not be satisfiable in $\mathcal{L}$, though there will be an extension $\mathcal{L}+$ of $\mathcal{L}$ such that $\Lambda$ is satisfiable with respect to the state-descriptions, not of $\mathcal{L}$, but of $\mathcal{L}+8$

${ }^{8}$ This is a known feature of substitutional quantification. See for instance Dunn and Belnap 1968. It 
Call a set $\Lambda$ of sentences of $\mathcal{L}, F C$-consistent (or simply consistent) iff there are no $\alpha_{1}, \ldots, \alpha_{\mathrm{n}} \in \Lambda$ such that $\vdash_{\mathrm{FC}} \sim\left(\alpha_{1} \wedge \ldots \wedge \alpha_{\mathrm{n}}\right)$. Suppose $\Lambda$ is a consistent set of sentences of a language $\mathcal{L}$. A set $\Gamma$ of sentences is maximal in $\mathcal{L}$ iff for every sentence $\alpha$ of $\mathcal{L}$, either $\alpha \in \Gamma$ or $\sim \alpha \in \Gamma$. Since FC is finitary $\Lambda$ will still be consistent in $\mathcal{L}^{+}$, where $\mathcal{L}^{+}$ contains infinitely many constants not in $\mathcal{L}$, and therefore not in $\Lambda$. The presence of I3 requires a change in what is called on p. 257 of Hughes and Cresswell 1996 the $\forall$-property. In a language without constants a set $\Delta$ has $\forall$-property iff for every wff in $\mathcal{L}$ of the form $\forall x \alpha$ there is some variable $y$ such that $\alpha[y / x] \supset \forall x \alpha \in \Delta$, and the standard technique for giving a consistent set the $\forall$-property, as for instance described in theorem 14.1 on p. 258 of Hughes and Cresswell 1996, has as a consequence that $y$ does not occur in $\alpha$. However, I3 makes the sentence $a \neq b \supset \forall x x \neq b$ inconsistent where $a$ is any constant distinct from $b$, and therefore inconsistent where $a$ is not in $x \neq b$. For that reason we shall introduce the Carnapian $\forall$-property, or $\forall_{\mathrm{C}}$-property:

Definition. $\forall_{\mathrm{C}}$-prop. $A$ set $\Delta$ of sentences of a language $\mathcal{L}$ of $F C$ has the $\forall_{\mathrm{C}}$-property in $\mathcal{L}$ iff for every sentence $\forall x \alpha$ in $\mathcal{L}$ there are constants a and $b_{1}, \ldots, b_{n}$ in $\mathcal{L}$ such that $\alpha[a / x] \supset \forall x\left(\left(x \neq b_{1} \wedge \ldots \wedge x \neq b_{-} n\right) \supset \alpha\right) \in \Delta$.

Lemma 7. Suppose $\Gamma$ is a maximal-consistent set of sentences with the $\forall_{\mathrm{C}}$-property, and suppose that $\forall x \alpha$ is a sentence such that $\alpha[a / x]$ is in $\Gamma$ for every constant a of $\mathcal{L}$. Then $\forall x \alpha \in \Gamma$.

Proof. If $\Gamma$ has the $\forall_{\mathrm{C}}$-property then there is some $a$, such that for some $b_{1}, \ldots, b_{\mathrm{n}}, \alpha[a / x] \supset$ $\left.\left.\forall x\left(x \neq b_{1} \wedge \ldots \wedge x \neq b_{n}\right) \supset \alpha\right)\right) \in \Gamma$. So, if $\alpha[c / x] \in \Gamma$ for every constant $c$, then in particular, $\alpha\left[b_{\mathrm{i}} / x\right] \in \Gamma$ for every $1 \leq \mathrm{i} \leq \mathrm{n}$. So, since $\Gamma$ is maximal-consistent, $\alpha\left[b_{1} / x\right] \wedge \ldots \wedge \alpha\left[b_{\mathrm{n}} / x\right]$ $\wedge \forall x\left(\left(x \neq b_{1} \wedge \ldots \wedge x \neq b_{\mathrm{n}}\right) \supset \alpha\right) \in \Gamma$, and so, by theorem $3, \forall x \alpha \in \Gamma$.

For a Carnapian language we may define the (ordinary) $\forall$-property of a set $\Delta$ of sentences to hold when, for every sentence $\forall x \alpha$, there is some constant $a$ such that $\alpha[a / x] \supset$ $\forall x \alpha \in \Delta$. From lemma 7 we have the following:

Corollary 8. If $\Gamma$ is a maximal-consistent set with the $\forall{ }_{\mathrm{C}}$-property then $\Gamma$ has the $\forall$ property.

The case of $a \neq b \supset \forall x x \neq b$ shews that in some cases the $\forall$-property requires a constant already occurring in $\alpha$. In this case we require $b \neq b \supset \forall x x \neq b$.

Theorem 9. Where $\Lambda$ is an $F C$-consistent set of sentences of a language $\mathcal{L}$ then there is

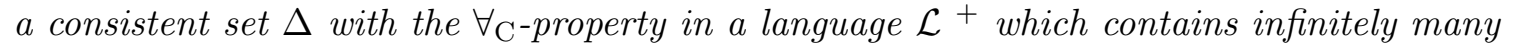
constants not in $\mathcal{L}$, such that $\Lambda \subseteq \Delta$

entails a failure of compactness in the sense that, although every finite subset of a set $\Lambda$ of sentence of a language $\mathcal{L}$ may be satisfied by a state description for $\mathcal{L}, \Lambda$ itself may not, though it will be satisfiable in a language with infinitely constants not in $\mathcal{L}$. The difference between the present completeness proof and more standard ones is that in the present case, though not in the standard case, the extension of the language is effectively accompanied by an extension of the domain.

Australasian Journal of Logic (11) 2014, Article no. 3 
Proof. Theorem 9 is proved by specifying a sequence $\Delta_{0}, \Delta_{1}, \ldots, \Delta_{\mathrm{n}}, \ldots$ defined as follows. Assume that there is an enumeration of wff of $\mathcal{L}$ each of which contains just one free variable, so that $\alpha_{\mathrm{n}}$ contains free $x_{\mathrm{n}}$, and so on. $\Delta_{0}$ is $\Lambda$, and, for $1 \leq n, \Delta_{\mathrm{n}}$ is $\Delta_{\mathrm{n}-1} \cup\left\{\alpha_{\mathrm{n}}\left[a / x_{\mathrm{n}}\right] \supset\right.$ $\left.\forall x_{\mathrm{n}}\left(\left(x_{\mathrm{n}} \neq b_{1} \wedge \ldots \wedge x_{\mathrm{n}} \neq b_{\mathrm{k}}\right) \supset \alpha\right)\right\}$, where $a$ is the first constant not already occurring in $\alpha$ or in any member of $\Delta_{\mathrm{n}-1}$ and $b_{1}, \ldots, b_{\mathrm{k}}$ are constants such that $\Delta_{\mathrm{n}-1} \cup\left\{\alpha_{\mathrm{n}}\left[a / x_{-} \mathrm{n}\right] \supset\right.$ $\left.\left.\forall x \_n\left(x_{\mathrm{n}} \neq b_{1} \wedge \ldots \wedge x_{\mathrm{n}} \neq b_{\mathrm{k}}\right) \supset \alpha\right)\right\}$ is consistent. For this to succeed it needs to be shewn that such a $\Delta_{\mathrm{n}}$ exists. If not, then

(i) For every $b_{1}, \ldots, b_{k}$, there are $\beta_{1}, \ldots, \beta_{m} \in \Delta_{n-1}$ such that $\vdash_{\mathrm{FC}}\left(\beta_{1} \wedge \ldots \wedge \beta_{m}\right) \supset \sim\left(\alpha\left[a / x_{n}\right] \supset \forall x_{n}\left(\left(x_{n} \neq b_{1} \wedge \ldots \wedge x_{n} \neq b_{k}\right) \supset \alpha\right)\right)$.

From (i) we have that there are $\gamma_{1}, \ldots, \gamma_{i} \in \Delta_{n-1}$ such that

$$
\text { (ii) } \vdash_{\mathrm{FC}}\left(\gamma_{1} \wedge \ldots \wedge \gamma_{\mathrm{i}}\right) \supset \alpha\left[a / x_{\mathrm{n}}\right]
$$

and so, by theorem 2 , since $a$ does not occur in $\Delta_{\mathrm{n}-1}$, there are constants $c_{1}, \ldots, c_{\mathrm{h}}$ such that

$$
\text { (iii) } \vdash_{\mathrm{FC}}\left(\gamma_{1} \wedge \ldots \wedge \gamma_{\mathrm{i}}\right) \supset \forall x_{\mathrm{n}}\left(\left(x_{\mathrm{n}} \neq c_{1} \wedge \ldots \wedge x_{\mathrm{n}} \neq c_{\mathrm{h}}\right) \supset \alpha\right) .
$$

Now (i) holds for every $b_{1}, \ldots, b_{k}$ and so, in particular for $c_{1}, \ldots, c_{h}$, and so there are $\delta_{1}, \ldots, \delta_{j} \in$ $\Delta_{n-1}$ such that

$$
\text { (iv) } \vdash_{\mathrm{FC}}\left(\delta_{1} \wedge \ldots \wedge \delta_{j}\right) \supset \sim \forall x_{n}\left(\left(x_{n} \neq c_{1} \wedge \ldots \wedge x_{n} \neq c_{h}\right) \supset \alpha\right) .
$$

and so

$$
(\mathrm{v}) \vdash_{\mathrm{FC}}\left(\gamma_{1} \wedge \ldots \wedge \gamma_{\mathrm{i}} \wedge \delta_{1} \wedge \ldots \wedge \delta_{\mathrm{j}}\right) \supset \sim \forall x_{\mathrm{n}}\left(\left(x_{\mathrm{n}} \neq c_{1} \wedge \ldots \wedge x_{\mathrm{n}} \neq c_{\mathrm{h}}\right) \supset \alpha\right) .
$$

But from (iii) we have

$$
\text { (vi) } \vdash_{\mathrm{FC}}\left(\gamma_{1} \wedge \ldots \wedge \gamma_{\mathrm{i}} \wedge \delta_{1} \wedge \ldots \wedge \delta_{\mathrm{j}}\right) \supset \forall x_{\mathrm{n}}\left(\left(x_{\mathrm{n}} \neq c_{1} \wedge \ldots \wedge x_{\mathrm{n}} \neq c_{\mathrm{h}}\right) \supset \alpha\right) .
$$

which with (v) yields

$$
\text { (vii) } \vdash_{\mathrm{FC}} \sim\left(\gamma_{1} \wedge \ldots \wedge \gamma_{i} \wedge \delta_{1} \wedge \ldots \wedge \delta_{j}\right)
$$

in which case $\Delta_{n-1}$ would be inconsistent. Let $\Delta$ be the union of all the $\Delta_{n} \mathrm{~s} . \Delta$ is consistent for the usual reasons. Further $\Delta$ has the $\forall_{\mathrm{C}}$-property in $\mathcal{L}{ }^{+}$.

Finally let $\Gamma$ be a maximal-consistent set containing $\Delta$. By corollary 8 , theorem 9 establishes that $\Gamma$ has the $\forall$-property.

Now let $s_{\Gamma}$, be the state-description which consists of $\varphi a_{1} \ldots a_{\mathrm{n}} \in s_{\Gamma}$ if $\varphi a_{1} \ldots a_{\mathrm{n}} \in \Gamma$, and $\sim \varphi a_{1} \ldots a_{n} \in s_{\Gamma}$ if $\sim \varphi a_{1} \ldots a_{n} \in \Gamma$.

Theorem 10. $s_{\Gamma} \models \alpha$ iff $\alpha \in \Gamma$. 
Proof. The result is defined for $\varphi a_{1} \ldots a_{n}$, and is obvious for $\perp$. If $s_{\Gamma}=a=b$, then these are the same constant, and from I1 we then have $a=b \in \Gamma$. If $s_{\Gamma}=a \neq b$, then these are distinct constants, and from I3 we have $a \neq b \in \Gamma$. The induction for $\perp$ and $\supset$ is standard. Suppose $s_{\Gamma}=\forall x \alpha$. Then there is a constant $a$ such that $s_{\Gamma}=\alpha[a / x]$. So, by the induction hypothesis $\alpha[a / x] \notin \Gamma$, and therefore, by $\forall 1 b, \forall x \alpha \notin \Gamma$. Suppose $\forall x \alpha \notin \Gamma$. Since $\Gamma$ has the $\forall$-property there is some $a$ such that $\alpha[a / x] \supset \forall x \alpha$. But then $\alpha[a / x] \notin \Gamma$, and so, by the induction hypothesis, $s_{\Gamma}=\alpha[a / x]$ and so $s_{\Gamma}=\forall x \alpha$.

Completeness follows in the usual way.

The main interest of Carnap 1946 is not, however, ordinary non-modal predicate logic. The purpose of Carnap's article is to study modal logic. If $\mathcal{L}$ is a language of ordinary firstorder logic we may extend $\mathcal{L}$ to obtain a language $\mathcal{L}_{\mathrm{M}} \cdot \mathcal{L}_{\mathrm{M}}$ is simply the modal predicate language which extends $\mathcal{L}$ by the addition of the (monadic) necessity operator $\square$, and the formation rule that if $\alpha$ is a wff then so is $\square \alpha$. The idea behind Carnap's account of modality is that $\square$ (which he writes as $N^{9}$ ) is to be interpreted in such a way that $\square \alpha$ is to be true iff $\alpha$ is valid - or, as Carnap puts it on p. 54, iff $\alpha$ is L-true. We shall make this precise as follows. Where $s$ is a state-description $[\mathrm{V} \varphi]-[\mathrm{V} \forall]$ shew how to extend $\models$ to all LPC wff. To extend $\models$ to all wff of $\mathcal{L}_{\mathrm{M}}$ in accordance with D9-5a (p. 54) we add the condition

$$
[\mathrm{V} \square \mathrm{MFL}] s \models \square \alpha \text { iff } s^{\prime} \models \alpha \text { for every state description } s^{\prime}
$$

Say that a wff is $C$-valid iff it is true in every state-description, when $\models$ satisfies $[\mathrm{V} \varphi]-[\mathrm{V} \forall]$ and $[\mathrm{V} \square \mathrm{MFL}]$. One result is that if $\alpha$ is $\mathrm{S} 5$-valid then $\alpha$ is $\mathrm{C}$-valid. However (quantified) S5 is not complete for C-validity. This is because, where $\varphi a$ is an atomic wff, $\sim \square \varphi a$ is C-valid even though it is not a theorem of S5 - and similarly with any atomic wff. Carnap specifies an axiomatic system of modal predicate logic, which he calls MFC. His first set of axioms (p. 54) is simply a basis sufficient for S5:

LPC $_{\square}$ Where $\alpha$ is one of the LPC axioms PC-I3 then both $\alpha$ and $\square \alpha$ are axioms of MFC. ${ }^{10}$

$$
\begin{aligned}
& \mathbf{K} \square \forall(\square(\alpha \supset \beta) \supset(\square \alpha \supset \square \beta)) \\
& \mathbf{T} \forall(\square \alpha \supset \alpha) \\
& \mathbf{5} \square \forall(\square \alpha \vee \square \sim \square \alpha)
\end{aligned}
$$

\footnotetext{
${ }^{9}$ The symbol $\square$ for necessity was devised by F.B. Fitch in 1945, and first appeared in print in Barcan 1946. I only met Carnap once, in 1970, shortly before his death. I asked him whether he or anyone else he knew of had ever used $L$ for a necessity operator before Feys 1950, but he could not recall any information about that. (I find it convenient to use $\square$ for logical necessity, which satisfies S5, and $L$ for the kind of necessity formalised by weaker modal systems.)

${ }^{10}$ Carnap separates this into a schema (D10-1a) which guarantees that PC is part of the basis, and then in D10-1, e to $\mathrm{k}$, repeats our $\forall^{\supset}$-I3.
}

Australasian Journal of Logic (11) 2014, Article no. 3 


$$
\begin{aligned}
\mathbf{B F}_{\mathrm{C}} \square \forall(\square \forall x \alpha \supset \forall x \square \alpha) \\
\text { BF } \square \forall(\forall x \square \alpha \supset \square \forall x \alpha)^{11}
\end{aligned}
$$

(A straightforward induction on proofs establishes the rule of necessitation, that if $\alpha$ is a theorem, so is $\square \alpha .{ }^{12}$ ) Carnap then includes some non-standard axioms, which shew that he is attempting to axiomatise $\mathrm{C}$-validity. These are what Carnap calls 'Assimilation', 'Variation and Generalization' and 'Substitution for Predicates'. (Carnap 1946, p. 54f.) In our notation these can be expressed as follows:

Ass $\square \forall x \forall y \forall z_{1} \ldots \forall z_{\mathrm{n}}\left(\left(x \neq z_{1} \wedge \ldots \wedge x \neq z_{\mathrm{n}}\right) \supset(\square \alpha \supset \square \alpha[y / x])\right)$, where $\alpha$ contains no free variables other than $x, y, z_{1}, \ldots, z_{\mathrm{n}}$, and no constants and no occurrences of $=$.

VG $\square \forall x \forall y \forall z_{1} \ldots \forall z_{\mathrm{n}}\left(\left(x \neq z_{1} \wedge \ldots \wedge x \neq z_{\mathrm{n}} \wedge y \neq z_{1} \wedge \ldots \wedge y \neq z_{\mathrm{n}}\right) \supset(\square \alpha \supset \square \alpha[y / x])\right.$, where $\alpha$ contains no free variables other than $x, y, z_{1}, \ldots, z_{\mathrm{n}}$, and no constants.

SP $\square \forall(\square \alpha \supset \square \beta)$, where $\alpha$ is a non-modal wff, and $\beta$ is obtained from $\alpha$ by uniform substitution of a complex expression for a predicate.

None of these axiom schemata is easy to process, but it is not difficult to see what the simplest instances would look like. A very simple instance, which is of both Ass and VG is

$$
\operatorname{Ass}_{\varphi} \square \forall x \forall y \forall z(x \neq z \supset(\square \varphi x y z \supset \square \varphi y y z))
$$

To establish the validity of $\operatorname{Ass}_{\varphi}$ it is sufficient to shew that if $a$ and $c$ are distinct constants then $\square \varphi a b c \supset \square \varphi b b c$ is valid. This is trivially so, since there is some $s$ such that $s=\varphi a b c$, and therefore for every $s, s=\square \varphi a b c$, and so, for every $s, s \models \square \varphi a b c \supset \varphi b b c$. More telling is the case of SP. Let $\varphi$ be a one-place predicate and consider

$$
\mathbf{S P}_{\varphi} \square \forall x(\square \varphi x \supset \square(\varphi x \wedge \sim \varphi x))
$$

In this case $\alpha$ is $\varphi x$, while $\beta$ is $\varphi x \wedge \sim \varphi x$, so that, in Carnap's words, $\beta$ 'is formed from $\alpha$ by replacing every atomic matrix containing $\varphi$ by the current substitution form of $\beta$ '.

\footnotetext{
${ }^{11}$ This principle is Carnap's T9-2 on p. 54 of Carnap 1946. The current name, 'Barcan Formula', comes from an equivalent formula first introduced in Barcan 1946, p. 2, Axiom 11. BF appears to have been first called the 'Barcan Formula' in Prior 1956, p. 60, where it is shewn derivable in S5. Even though Carnap includes $\mathrm{BF}_{\mathrm{C}}$ as an axiom, it is in fact derivable from the rest of the basis using only principles of non-modal logic together with $\mathrm{LPC}_{\square}$ and $\mathrm{K}$. By $\forall 1 \mathrm{~b}$ we have $\square(\forall x \alpha \supset \alpha[a / x])$ and so, by $\mathrm{K}, \square \forall x \alpha \supset \square \alpha[a / x]$ for every constant $a$. So choose some $a$ not in $\square \forall x \alpha$. Then, by theorem 2 , we have that there are $b_{1}, \ldots, b_{n}$ such that $\left.\vdash \square \forall x \alpha \supset \forall x\left(x \neq b_{1} \wedge \ldots \wedge x \neq b_{n}\right) \supset \square \alpha\right)$. But we also have $\square \forall x \alpha \supset \square \alpha\left[b_{i} / x\right]$ for $1 \leq i \leq n$, and so, by theorem 3 , we have $\square \forall x \alpha \supset \forall x \square \alpha$.

${ }^{12}$ The only non-trivial case is the axiom T, where we require $\square \forall(\square \alpha \supset \alpha)$, which is Carnap's theorem T4-1u on p. 42.
}

Australasian Journal of Logic (11) 2014, Article no. 3 
Thus we have $\vdash \sim \square(\varphi a \wedge \sim \varphi a)$, and so $\vdash \sim \square \varphi a .^{13}$

A completeness result for C-validity is not possible. For, although Carnap appears to have it in mind to axiomatise C-validity, it is easy to see that it is not recursively axiomatisable. For, by [V $\square$ MFL], where $\alpha$ is any LPC wff, $\alpha$ is not LPC-valid iff $\sim \square \alpha$ is $\mathrm{C}$-valid, and so, if C-validity were axiomatisable then LPC would be decidable. ${ }^{14}$ There is a hint on p. 57 that Carnap may have recognised this. He is certainly aware that the kind of reduction to normal form, with which he achieves the completeness of propositional S5, is unavailable in the predicate case, since it would lead to the decidability of LPC.

It is however worth looking at a modification of Carnap's semantics which brings it into line with the current indexical semantics for modal logic.

Let $\Sigma$ be a class of state-descriptions, where each $s \in \Sigma$ satisfies [V $\varphi$ ]-[V $\forall$ ], and [V $\left.\square_{\mathrm{MFL}}\right]$ is replaced by

$\left[\mathrm{V}_{\mathrm{MFL}_{\Sigma}}\right] s=\square \alpha$ iff $s^{\prime} \models \alpha$ for every $s^{\prime} \in \Sigma$

Say that a sentence $\alpha$ is valid in $\Sigma$ iff $s=\alpha$ for every $s \in \Sigma$, and say that $\alpha$ is $\Sigma^{+}$-valid iff $\alpha$ is valid in every $\Sigma$. With this change it is easy to see that $\sim \square \varphi a$ is not $\Sigma^{+}$-valid, since one need only consider, for instance, a class $\Sigma$ whose only member is a state-description $s$, where $s$ contains $\varphi a$. In fact one can prove that a Carnapian version of the standard axiomatic basis for predicate S5 is complete for this semantics. To avoid confusion with Carnap's MFC, which does contain the non-standard axioms, I shall refer to the system for which completeness is to be proved as $\mathrm{FC}+\mathrm{S} 5$. That is to say, completeness can be proved with respect to the semantics based on an arbitrary set of state-descriptions, for the basis which consists of $\mathrm{LPC}_{\square-\mathrm{BF}}$, but not containing Ass, VG or SP.

It is easy to establish that all the axioms of $\mathrm{FC}+\mathrm{S} 5$ are $\Sigma^{+}-$valid, and that MP preserves $\Sigma^{+}-$validity. For completeness we need some standard results from modal logic. Where $\Lambda$ is a set of sentences let $\mathrm{L}^{-}(\Lambda)$ denote the set consisting precisely of every sentence $\beta$ for which $\square \beta$ is in $\Lambda$. I.e., $\mathrm{L}^{-}(\Lambda)=\{\beta: \square \beta \in \Lambda\}$.

Lemma 11. Let $\Lambda$ be a set of sentences containing $\sim \square \alpha$. Then $L^{-}(\Lambda) \cup\{\sim \alpha\}$ is FC+S5consistent. ${ }^{15}$

The following theorem should be compared with theorem 14.2 on p. 259 of Hughes and Cresswell 1996.

\footnotetext{
${ }^{13}$ I.e., where $\beta$ is $\varphi x \wedge \sim \varphi x$, it replaces $\alpha$ 's $\varphi x$. If $\alpha$ had been more complex and contained $\varphi y$ as well as $\varphi x$, then the replacement would have given $\varphi y \wedge \sim \varphi y$, and so on, where care needs to be taken to prevent any free variable being bound as a result of the replacement. Substitution of complex expressions for predicates is a complex business. For a hint of some of the complexities of substitution rules for predicates see Church 1956 p. 289f. Carnap spells out the rule for substitution for predicates in D10-1p on p. 55.

${ }^{14}$ Although that is so it has not always been regarded as a criticism. Cocchiarella 1975 for one welcomes the various unusual features of Carnap's logic. (See for instance pp. 45 and 54f.)

${ }^{15}$ See lemma 6.4 on p. 117 of Hughes and Cresswell, 1996.
} 
Theorem 12. If $\Gamma$ is a maximal-consistent set of sentences in some language (say $\mathcal{L}^{+}$) of modal predicate logic, and $\Gamma$ has the $\forall$-property in $\mathcal{L}^{+}$, and $\alpha$ is a sentence such that $\square \alpha \notin \Gamma$, then there is a consistent set $\Delta$ of sentences of $\mathcal{L}^{+}$with the $\forall$-property such that $L^{-}(\Gamma) \cup\{\sim \alpha\} \subseteq \Delta$.

Proof. We define a sequence of sentences $\gamma_{0}, \gamma_{1}, \gamma_{2}, \ldots$ etc. $\gamma_{0}$ is $\sim \alpha$. Given $\gamma_{n}$ we define $\gamma_{n+1}$ as follows. Let $\forall x \delta$ be the $\mathrm{n}+1$ th sentence of that form and let $a$ be the first constant such that

$$
\text { (i) } \mathrm{L}^{-}(\Gamma) \cup\left\{\gamma_{\mathrm{n}} \wedge(\delta[a / x] \supset \forall x \delta)\right\} \text { is consistent. }
$$

Let $\gamma_{\mathrm{n}+1}$ be $\gamma_{\mathrm{n}} \wedge(\delta[a / x] \supset \forall x \delta)$. Since $\gamma_{0}$ is $\sim \alpha, \mathrm{L}^{-}(\Gamma) \cup\left\{\gamma_{0}\right\}$ is consistent from lemma 11. We shew that provided $\mathrm{L}^{-}(\Gamma) \cup\left\{\gamma_{\mathrm{n}}\right\}$ is consistent there will always be an $a$ which satisfies (i). Suppose there were not. Then, for every constant $a$, there will exist some $\left\{\beta_{1}, \ldots, \beta_{\mathrm{k}}\right\} \subseteq \mathrm{L}^{-}(\Gamma)$ such that

$$
\text { (ii) } \vdash\left(\beta_{1} \wedge \ldots \wedge \beta_{\mathrm{k}}\right) \supset\left(\gamma_{\mathrm{n}} \supset \sim(\delta[a / x] \supset \forall x \delta)\right)
$$

so, by principles of $\mathrm{S} 5^{16}$

$$
\text { (iii) } \vdash\left(\square \beta_{1} \wedge \ldots \wedge \square \beta_{\mathrm{k}}\right) \supset \square\left(\gamma_{\mathrm{n}} \supset \sim(\delta[a / x] \supset \forall x \delta)\right)
$$

But $\Gamma$ is maximal consistent and $\square \beta_{1}, \ldots, \square \beta_{\mathrm{k}} \in \Gamma$, and so

$$
\text { (iv) } \square\left(\gamma_{\mathrm{n}} \supset \sim(\delta[a / x] \supset \forall x \delta)\right) \in \Gamma .
$$

Now (iv) holds for every constant $a$, and $\Gamma$ has the $\forall$-property. What this means is this. Let $z$ be some variable not occurring in $\delta$ or in $\gamma_{\mathrm{n}}$, and consider $\forall z \square\left(\gamma_{\mathrm{n}} \supset \sim(\delta[z / x] \supset \forall x \delta)\right)$. Since $\Gamma$ has the $\forall$-property there will be a constant $a$ such that

$$
\text { (v) } \square\left(\gamma_{\mathrm{n}} \supset(\sim(\delta[a / x] \supset \forall x \delta)) \supset \forall z \square\left(\gamma_{\mathrm{n}} \supset \sim(\delta[z / x] \supset \forall x \delta)\right) \in \Gamma\right.
$$

and so, by (iv)

$$
\text { (vi) } \forall z \square\left(\gamma_{n} \supset \sim(\delta[z / x] \supset \forall x \delta)\right) \in \Gamma
$$

and so, by BF,

$$
\text { (vii) } \square \forall z\left(\gamma_{n} \supset \sim(\delta[z / x] \supset \forall x \delta)\right) \in \Gamma
$$

Since $z$ does not occur in $\gamma_{n}$ or $\delta$ then by $\forall^{\supset}$ and VQ we have in $\Gamma$

$$
\text { (viii) } \square\left(\gamma_{n} \supset \forall z \sim(\delta[z / x] \supset \forall x \delta)\right)
$$

But by lemma 4

\footnotetext{
${ }^{16}$ See p. 117 of Hughes and Cresswell 1996.
} 


$$
\text { (ix) } \vdash \exists z(\delta[z / x] \supset \forall x \delta)
$$

But then $\square \sim \gamma_{n} \in \Gamma$ and so $\sim \gamma_{n} \in \mathrm{L}^{-}(\Gamma)$ which would make $\mathrm{L}^{-}(\Gamma) \cup\left\{\gamma_{n}\right\}$ inconsistent.

Now assume that $\Lambda$ is an $\mathrm{FC}+\mathrm{S} 5$-consistent set of sentences, and that $\Gamma$ is a maximalconsistent set of sentences with the $\forall_{\mathrm{C}}$-property constructed in accordance with the procedure of theorem 9. By corollary 8, $\Gamma$ has the $\forall$-property. Let $\Gamma^{+}$be the set of all maximal consistent sets $w$ with the $\forall$-property, such that $\mathrm{L}^{-}(\Gamma) \subseteq w$. The presence of $\mathbf{T}$ ensures that $\mathrm{L}^{-}(\Gamma) \subseteq \Gamma$, and therefore that $\Gamma \in \Gamma^{+}$. Let $\Sigma$ be the set of all state descriptions $s_{w}$ where, for $w \in \Gamma^{+}, s_{w}$ is the state-description which consists of $\varphi a_{1} \ldots a_{n} \in$ if $\varphi a_{1} \ldots a_{\mathrm{n}} \in w$, and $\sim \varphi a_{1} \ldots a_{\mathrm{n}} \in s_{w}$ if $\varphi a_{1} \ldots a_{\mathrm{n}} \notin w$. We then have the following analogue of theorem 10:

Theorem 13. For any $w \in \Gamma^{+}, s_{w} \models \alpha$ iff $\alpha \in w$

Proof. The inductive proof is as for theorem 10, except for the induction for $\square$. Suppose that $\square \beta \in w$. Then $\square \beta \in \Gamma$, since if not, by $\mathbf{5}, \square \sim \square \beta \in \Gamma$, and then $\sim \square \beta \in w$. But if $\square \beta \in \Gamma$ then $\beta \in w^{\prime}$ for every $w^{\prime} \in \Gamma^{+}$, and so, by the induction hypothesis, $s_{w^{\prime}}=\beta$ for every $s_{w^{\prime}} \in \Sigma$, and so $s_{w}=\square \beta$. If $\square \beta \notin w$ then $\sim \square \beta \in \Gamma$, since if $\square \beta \in \Gamma$ then $\square \square \beta \in \Gamma$ and then $\square \beta \in w$. But if $\sim \square \beta \in \Gamma$ then, by theorem 12, there is some $w^{\prime} \in \Gamma^{+}$such that $\beta \notin w^{\prime}$. So, by the induction hypothesis, $s_{w^{\prime}}=\beta$, and so $s_{w}=\square \beta$.

From theorem 13 it follows that the members of $\Lambda$ are true in $s_{\Gamma}$, and therefore, since $\Gamma \in \Gamma^{+}$, true in some $s \in \Sigma$, thus establishing the completeness of $\mathrm{FC}+\mathrm{S} 5$ with respect to $\Sigma^{+}$- validity.

Finally we shall revisit Ass-SP. As noted, neither they nor any other recursively specified axiom set is complete for C-validity. However, a rather trivial completeness proof is possible based on $\Sigma^{+}$-validity. MFC is FC + S5, with the addition of all instances of Ass-SP. Let a sentence $\alpha$ be MFC-valid iff $\alpha$ is valid in every class $\Sigma$ of state-descriptions on which every instance of Ass-SP is valid. It is trivial that MP preserves MFC-validity. ${ }^{17}$ So if $\vdash_{\mathrm{MFC}} \alpha$ then $\alpha$ is MFC-valid. If $\dashv_{\mathrm{MFC}} \alpha$ then, where $\Delta$ consists of all instances of Ass-SP, $\Delta \cup\{\sim \alpha\}$ is $\mathrm{FC}+\mathrm{S} 5$-consistent. So, according to theorem 13, there is a class $\Sigma$ of state descriptions such that, for some $s \in \Sigma$, every instance of Ass-SP is true at $s$ but $\alpha$ is not; which is just to say that $\alpha$ is not MFC-valid.

\section{Appendix: Proofs of theorems 2 and 3, and lemma 4:}

The proofs of the following three results have no theoretical interest, except to establish that they do indeed follow from Carnap's axioms, as presented in this paper. Where the

\footnotetext{
${ }^{17}$ Further, assuming the consistency of the part of set theory needed to define the class of all statedescriptions for a denumerable language, all of Ass-SP will be valid on that class, and so MFC will be consistent.
}

Australasian Journal of Logic (11) 2014, Article no. 3 
justification for a line of the proof is given by merely citing earlier lines of the proof the line is obtained from an instance of $\mathbf{P C}$ or $\forall \supset$ and applications of MP.

Theorem 2:

Proof. The theorem may be proved as follows. Assume

(1) $\alpha \supset \beta$

Then, by theorem 1 , there are $b_{1}, \ldots, b_{\mathrm{n}}$ such that

$$
\text { (2) } \forall x\left(\left(x \neq b_{1} \wedge \ldots \wedge x \neq b_{\mathrm{n}}\right) \supset(\alpha \supset \beta)[x / a]\right)
$$

But $a$ does not occur in $\alpha$ and so

$$
\text { (3) } \forall x\left(\left(x \neq b_{1} \wedge \ldots \wedge x \neq b_{\mathrm{n}}\right) \supset(\alpha \supset \beta[x / a])\right)
$$

So, by $\mathrm{PC}$ and $\forall^{\supset}$,

$$
\text { (4) } \left.\forall x \alpha \supset \forall x\left(\left(x \neq b_{1} \wedge \ldots \wedge x \neq b_{\mathrm{n}}\right) \supset \beta[x / a]\right)\right)
$$

But $\alpha$ is a sentence since $\alpha \supset \beta$ is a theorem, and so, by VQ

(5) $\alpha \supset \forall x \alpha$

(4)(5) PC

(6) $\left.\alpha \supset \forall x\left(\left(x \neq b_{1} \wedge \ldots \wedge x \neq b_{\mathrm{n}}\right) \supset \beta[x / a]\right)\right)$ 
Theorem 3:

Proof.

$$
\begin{array}{lll}
\mathrm{I} 1, \mathrm{I} 2 & (1) & \forall x\left(x=b_{\mathrm{i}} \supset b_{\mathrm{i}}=x\right)(1 \leq i \leq n) \\
\mathrm{I} 2, \forall 1 \mathrm{~b} & (2) & \forall x\left(b_{\mathrm{i}}=x \supset\left(\alpha\left[b_{\mathrm{i}} / x\right] \supset \alpha\right)\right) \\
(1)(2) & (3) & \forall x\left(x=b_{\mathrm{i}} \supset\left(\alpha\left[b_{\mathrm{i}} / x\right] \supset \alpha\right)\right) \\
(3) & (4) & \forall x\left(\alpha\left[b_{\mathrm{i}} / x\right] \supset\left(x=b_{\mathrm{i}} \supset \alpha\right)\right) \\
(4) & (5) & \forall x\left(\alpha\left[b_{\mathrm{i}} / x\right]\right) \supset \forall x\left(x=b_{\mathrm{i}} \supset \alpha\right) \\
(5) & (6) & \forall x\left(\left(\alpha\left[b_{1} / x\right] \wedge \ldots \wedge \alpha\left[b_{\mathrm{n}} / x\right]\right) \supset \forall x\left(\left(x=b_{1} \vee \ldots \vee x=b_{\mathrm{n}}\right) \supset \alpha\right)\right) \\
\mathrm{VQ} & (7) & \left(\alpha\left[b_{1} / x\right] \wedge \ldots \wedge\left[b_{\mathrm{n}} / x\right]\right) \supset \forall x\left(\alpha\left[b_{1} / x\right] \wedge \ldots \wedge \alpha\left[b_{\mathrm{n}} / x\right]\right) \\
(6)(7) & (8) & \left(\alpha\left[b_{1} / x\right] \wedge \ldots \wedge \alpha\left[b_{\mathrm{n}} / x\right]\right) \supset \forall x\left(\left(x=b_{1} \vee \ldots \vee x=b_{\mathrm{n}}\right) \supset \alpha\right) \\
\mathrm{PC} & (9) & \forall x\left(\left(\left(x=b_{1} \vee \ldots \vee x=b_{\mathrm{n}}\right) \supset \alpha\right) \supset\left(\left(\left(x \neq b_{1} \wedge \ldots \wedge x \neq b_{\mathrm{n}}\right) \supset \alpha\right) \supset \alpha\right)\right) \\
(9) \forall \supset & (10) & \left.\forall x\left(\left(x=b_{1} \vee \ldots \vee x=b_{\mathrm{n}}\right) \supset \alpha\right) \supset \forall x\left(\left(\left(x \neq b_{1} \wedge \ldots \wedge x \neq b_{\mathrm{n}}\right) \supset \alpha\right) \supset \alpha\right)\right) \\
(10) \forall \supset & (11) & \left.\forall x\left(\left(x=b_{1} \vee \ldots \vee x=b_{\mathrm{n}}\right) \supset \alpha\right)\right) \supset\left(\forall x\left(\left(x \neq b_{1} \wedge \ldots \wedge x \neq b_{\mathrm{n}}\right) \supset \alpha\right) \supset \forall x \alpha\right) \\
(8)(11) & (12) & \left(\alpha\left[b_{1} / x\right] \wedge \ldots \wedge \alpha\left[b_{\mathrm{n}} / x\right] \wedge \forall x\left(\left(x \neq b_{1} \wedge \ldots \wedge x \neq b_{\mathrm{n}}\right) \supset \alpha\right)\right) \supset \forall x \alpha
\end{array}
$$

Lemma 4:

PC

$\forall 1$ b PC Def $\exists$

Quine 1940 , p. $112,{ }^{*} 170$

(1) (2) (3) PC

PC

(5) $\forall \supset$

(6) PC Def $\exists$

(4) (7) PC
(1) $\forall x \alpha \supset(\alpha[a / x] \supset \forall x \alpha)$

(2) $(\alpha[a / x] \supset \forall x \alpha) \supset \exists y(\alpha[y / x] \supset \forall x \alpha)$

(3) $\forall y \alpha[y / x] \supset \forall x \alpha$

(4) $\forall y \alpha[y / x] \supset \exists y(\alpha[y / x] \supset \forall x \alpha)$

(5) $\forall y(\sim \alpha[y / x] \supset \forall x \alpha) \supset \alpha[y / x])$

(6) $\forall y \sim(\alpha[y / x] \supset \forall x \alpha) \supset \forall y \alpha[y / x]$

(7) $\sim \forall y \alpha[y / x] \supset \exists y(\alpha[y / x] \supset \forall x \alpha)$

(8) $\exists y(\alpha[y / x] \supset \forall x \alpha)$

\section{References}

[1] Barcan, (Marcus) R.C., 1946, A functional calculus of first order based on strict implication, The Journal of Symbolic Logic, 11, 1-16

[2] Beth, E.W., 1959, The Foundations of Mathematics, Amsterdam, North-Holland Publishing Company

[3] Berry, G. D. W., 1941 On Quine's Axioms of Quantification. The Journal of Symbolic Logic, Vol. 6, pp. 23-27

Australasian Journal of Logic (11) 2014, Article no. 3 
[4] Carnap, R., 1946, Modalities and quantification. The Journal of Symbolic Logic, 11, 33-64

[5] Carnap, R., 1947, Meaning and necessity, Chicago, University of Chicago Press (Second edition 1956, references are to the second edition.).

[6] Church, A., 1956, Introduction to mathematical logic Vol. I, Princeton, Princeton University Press

[7] Cocchiarella, N.B., 1975, Logical Atomism, Nominalism, and Modal Logic. Synthese, Vol 31, pp. 23-67.

[8] Dunn, J.M., and N.D. Belnap, 1968, The substitution interpretation of the quantifiers Noũs. Vol 2, pp. 177-185

[9] Feys, R., 1950, Les systèmes formalisés Aristotéliciennes, Revue Philosophique de Louvain, 48, 478-509

[10] Fine, K., 1983, The permutation principle in quantificational logic. Journal of Philosophical Logic, 12, 33-7

[11] Fitch, F. B. 1941, Closure and Quine's *101. The Journal of Symbolic Logic, Vol. 6, pp. $18-22$

[12] Gödel, K., 1930, Die Vollständigkeit der Axiome des logischen Fuktionkalkulüs, Monatshefte für Mathematik und Physik, vol 37, pp. 349-36

[13] Henkin, L. A., 1949, The completeness of the first-order functional calculus, The Journal of Symbolic Logic, 14, 159-66

[14] Hughes, G.E. and M.J. Cresswell, 1996, A New Introduction to Modal Logic, London, Routledge

[15] Leblanc, H., 1969, Three generalizations of a theorem of Beth's. Logique et Analyse, No 47 , pp. 205-220

[16] Leblanc, H., 1973, Semantic derivations. Truth, Syntax and Modality, Amsterdam, North-Holland Publishing Company, pp. 1-16

[17] Prior, A.N., 1956, Modality and quantification in S5, The Journal of Symbolic Logic, $21,60-2$

[18] Quine, W. V., 1940, Mathematical logic, New York, Norton. (Second edition, 1951. page references are to the reprinting by Harper Torchbooks in 1962.)

[19] Rogers, B., and K.F. Wehmeier, 2012, Tractarian first-order logic: identity and the N operator, The Review of Symbolic Logic, Vol 5, pp. 538-573

Australasian Journal of Logic (11) 2014, Article no. 3 
[20] Wittgenstein, L., 1922, Tractatus Logico-Philosophicus. (Translated by C.K. Ogden) London, Kegan Paul)

Australasian Journal of Logic (11) 2014, Article no. 3 